\title{
https://doi.org/10.46813/2020-130-041 ION ACOUSTIC INSTABILITY IN DUSTY PLASMA
}

\author{
Y.V. Susayev ${ }^{1}$, V.V. Olshansky ${ }^{1,2}$ \\ ${ }^{1}$ V.N. Karazin Kharkiv National University, Kharkiv, Ukraine; \\ ${ }^{2}$ Institute of Plasma Physics NSC "Kharkov Institute of Physics and Technology”, Kharkiv, Ukraine \\ E-mail: olshansky@kipt.kharkov.ua
}

The computer simulation results of the ion acoustic instability evolution in the dusty plasma are presented. The dusty plasma consists from electrons, ions, massive charged dust grains and neutral atoms. In the simulation the implicit PIC-code is used. To this code the implicit scheme of particles movement is applied, namely the direct implicit method of Langdon-Fridman. Realization of the algorithm is presented too.

PACS: 52.35.Qz; 52.65.Rr; 2.80.Pi;52.27.Lw

\section{INTRODUCTION}

It is known that the presence of dust in the plasma significantly affects the collective processes that take place in it. Dust can change both the spectra of oscillations and instabilities that exist in plasma without dust, and generate new branches of oscillations and new specific instabilities. The reason for this is that the presence of dust changes the characteristic spatiotemporal scales in the plasma. For example, the plasma frequency of the dust component of the plasma due to the large mass of dust grains is less than the ion plasma frequency by several orders of magnitude. As the charge mixture of the plasma changes, in particular, the dispersion of ion acoustic oscillations changes and a new low-frequency branch, namely dusty acoustic waves, appears.

One of the directions of modern research of dusty plasma is the study of instabilities that occur under the action of an external electromagnetic field of large amplitude. Among them are studies of the interaction between particles in plasma, forces acting on the dusty grains and low-frequency ion acoustic instability in dusty plasma, and so on. In this paper, the development of ion acoustic instability in dusty plasma is considered. For self-consistent modeling of low-frequency ion acoustic instability in dusty plasma, the "ParticleParticle Particle-Mesh" (P3M) model was used [1], and a corresponding kinetic code based on the PIC / MCC model was developed [2]. The choice of this model is due to the fact that although the method "particle-incell" (PIC) has proven its effectiveness in modeling plasma, it has a significant disadvantage for the study of dusty plasma. The spatial distinction in the PIC scheme is limited by the size of the spatial grid, which is usually of the order of the Debye radius. For a thermonuclear plasma, the size of the Debye radius of the tenth or hundredth of a millimeter. The size of the powders is in the micrometer range, i.e. much smaller than the size of the spatial grid. In PIC - models the plasma particles are represented by charged "clouds", which have the size of the cell mesh. This leads to an inaccurate presentaton of the interaction of particles when the distance between them becomes less than the size of the spatial grid. Thus, the force of interaction between particles at a small distance is very different from the Coulomb and goes to zero when the distance between the particles ISSN 1562-6016. BAHT. 2020. №6(130)

PROBLEMS OF ATOMIC SCIENCE AND TECHNOLOGY. 2020, № 6. Series: Plasma Physics (26), p. 41-46. decreases. To overcome this problem, the PIC-MCC collision operator [3] with a cross-section of collisions obtained in the analytical theory of "orbital motion limit" (OML) [4] could be used to describe collisions of plasma particles with powders. This model is fast enough, but unsuitable when the plasma is highly inhomogeneous and not Maxwellian.

The P3M model is a combination of the PIC model with the technique used in molecular dynamics (MD) and is suitable for modeling the effects associated with finite dust grain sizes. The plasma itself is modeled in a self-consistent manner. This means that the long-range interaction of dust with charged plasma particles is described in accordance with the PIC formalism. At the same time, for plasma particles that are at a distance less than the Debye radius from the dust grains, the interaction force is calculated according to the direct particle-particle scheme, which uses the exact Coulomb potential. The interaction between the particles of the plasma in the same region is calculated by the PIC scheme. To reduce the computer time for calculating the direct particle-particle interaction, the so-called "chain grid" is additionally introduced [1]. On the scales of the order of the size of the dust grains, the motion of the plasma particles is calculated with a smaller time step than in areas far from them. The typical time step in the PIC circuit is of the order of $10^{-11} \mathrm{~s}$. At the same time, near the dust grains, the characteristic time step is about $10^{-13} \mathrm{~s}$. Plasma particles that cross the boundary surface of the powder are considered to be absorbed. The charge of the powders is renewed at each time step.

\section{IMPLICIT SCHEME OF INTEGRATION OF EQUATIONS OF MOTION}

Due to the huge difference in the masses of electrons and ions, not to mention the comparison with the masses of the dust grains, the code uses an implicit scheme for integrating the equations of motion, namely the direct implicit method of Langdon and Friedman. The essence of this method is that recursive filtering of the electric field suppresses high-frequency modes. Therefore, this scheme allows you to use a large time step, limited only by the value of the characteristic time of the problem.

The following implicit finite-difference scheme is used to integrate the equations of motion in a cylindrical coordinate system. 


$$
\left\{\begin{array}{rrr}
\mathbf{v}_{n+1 / 2}-\mathbf{v}_{n-1 / 2}= & \mathbf{a}_{n} \Delta t+\beta\left(\mathbf{v}_{n+1 / 2}+\mathbf{v}_{n-1 / 2}\right) \times \mathbf{B}\left(\mathbf{x}_{n}\right), \\
\mathbf{x}_{n+1}= & \mathbf{x}_{n}+\mathbf{v}_{n+1 / 2} \Delta t, \\
\mathbf{a}_{n}= & \frac{1}{2}\left(\frac{q}{m} \mathbf{E}_{n+1}\left(\mathbf{x}_{n+1}\right)+\mathbf{a}_{n-1}\right),
\end{array}\right.
$$

where $\beta=\frac{q \Delta t}{2 m c}$. The first equation in system (1) is solved with respect to velocity $\mathbf{v}_{n+1 / 2}$ in the next time step.

$$
\begin{aligned}
& \mathbf{v}_{n+1 / 2}=\left(1+\beta^{2} B_{n}^{2}\right)^{-1}\left[\left(1-\beta^{2} B_{n}^{2}\right) \mathbf{v}_{n-1 / 2}+2 \beta \mathbf{v}_{n-1 / 2} \times \mathbf{B}_{n}+\right. \\
& \left.+2 \beta^{2}\left(\mathbf{v}_{n-1 / 2} \cdot \mathbf{B}_{n}\right) \mathbf{B}_{n}+\Delta t\left(\mathbf{a}_{n}+\beta \mathbf{a}_{n} \times \mathbf{B}_{n}+\beta^{2}\left(\mathbf{a}_{n} \cdot \mathbf{B}_{n}\right) \mathbf{B}_{n}\right)\right] .
\end{aligned}
$$

Members who depend only on the time step $t_{n}$ and are even more advanced in time are separated. Then equation (2) takes the form

$$
\mathbf{v}_{n+1 / 2}=\frac{\Delta t q}{2 m}\left(1+\beta^{2} B_{n}^{2}\right)^{-1}\left(\mathbf{E}_{n+1}+\beta \mathbf{E}_{n+1} \times \mathbf{B}_{n}+\beta^{2}\left(\mathbf{E}_{n+1} \cdot \mathbf{B}_{n}\right) \mathbf{B}_{n}\right)+\widetilde{\mathbf{v}},
$$

where $\tilde{\mathbf{v}}$ is an intermediate velocity

$$
\begin{aligned}
& \tilde{\mathbf{v}}=\left(1+\beta^{2} B_{n}^{2}\right)^{-1}\left[\left(1-\beta^{2} B_{n}^{2}\right) \mathbf{v}_{n-1 / 2}+2 \beta \mathbf{v}_{n-1 / 2} \times \mathbf{B}_{n}+\right. \\
& \left.+2 \beta^{2}\left(\mathbf{v}_{n-1 / 2} \cdot \mathbf{B}_{n}\right) \mathbf{B}_{n}+\Delta t / 2 \cdot\left(\mathbf{a}_{n-1}+\beta \mathbf{a}_{n-1} \times \mathbf{B}_{n}+\beta^{2}\left(\mathbf{a}_{n-1} \cdot \mathbf{B}_{n}\right) \mathbf{B}_{n}\right)\right] .
\end{aligned}
$$

Corresponding intermediate positions of the particles

$$
\widetilde{\mathbf{x}}=\mathbf{x}_{n}+\widetilde{\mathbf{v}} \Delta t
$$

Assuming axial symmetry, the electric and external magnetic fields will depend on only two cylindrical coordinates $\{\mathrm{z}, \mathrm{r}\}$ and will have only two components $\mathbf{E}=\left\{E_{z}, E_{r}\right\}, \mathbf{B}=\left\{B_{z}, B_{r}\right\}$. The motion of particles in a time step $t_{n}$ is determined by three components of velocity $v_{r}, v_{\theta}, v_{z}$. Although the PIC particle does not have an azimuthal coordinate, it has an azimuthal velocity component that describes the rotation of all real plasma particles containing one PIC particle around the $z$ axis as a whole. Similar to [5], an auxiliary Cartesian coordinate system $\left\{\mathrm{x}_{1}, \mathrm{x}_{2}, \mathrm{x}_{3}\right\}$ is introduced to move a particle in time by one step.

This system is oriented in such a way that its axis $\mathrm{x}_{1}$ has some direction perpendicular to the cylindrical axis, at the same time the axis $x_{3}$ and the beginning of the auxiliary coordinate system coincide with the $z$-axis of the main cylindrical system. Uncertainty arises due to the fact that the PIC particle has no azimuthal coordinate. Regarding the auxiliary Cartesian coordinate system, the velocity and acceleration of a particle under the action of a field at a time step $t_{n}$ are

$$
\begin{aligned}
& x_{n}=\left[x_{3, n}, x_{1, n}\right]^{T}=\left[z_{n}, r_{n}\right]^{T}, \\
& v_{n}=\left[v_{1, n}, v_{2, n}, v_{3, n}\right]^{T}=\left[v_{r, n}, v_{\theta, n}, v_{z, n}\right]^{T}, \\
& a_{n}=\left[a_{3, n}, a_{1, n}\right]^{T}=\left[a_{z, n}, a_{r, n}\right]^{T} .
\end{aligned}
$$

Appropriate Cartesian components of electric and magnetic fields will be

$$
\begin{aligned}
& E_{n}=\left[E_{3, n}, E_{1, n}\right]^{T}=\left[E_{z, n}, E_{r, n}\right]^{T}, \\
& B_{n}=\left[B_{3, n}, B_{1, n}\right]^{T}=\left[B_{z, n}, B_{r, n}\right]^{T} .
\end{aligned}
$$

Let us denote the matrix by the symbol $\mathrm{B}_{\mathrm{n}}$

$$
\mathbf{B}_{n}=\left[\begin{array}{ccc}
0 & B_{3, n} & 0 \\
-B_{3, n} & 0 & B_{1, n} \\
0 & -B_{1, n} & 0
\end{array}\right]
$$

and rewrite equations (4) and (5) in matrix notation

$$
\begin{aligned}
& \tilde{\mathbf{v}}=\mathbf{T}_{n} \mathbf{a}_{n-1}+\mathbf{R}_{n} \mathbf{v}_{n-1 / 2}, \\
& \widetilde{\mathbf{x}}=\mathbf{x}_{n}+\mathbf{P}_{23} \tilde{\mathbf{v}} \Delta t .
\end{aligned}
$$

In equations (6) and (7) the following symbols are introduced

$$
\begin{aligned}
& \mathbf{T}_{n}=\frac{\Delta t}{2}\left(\mathbf{I}-\mathbf{B}_{\beta n}\right)^{-1} \mathbf{P}_{32}, \mathbf{B}_{\beta n}=\beta \mathbf{B}_{n}, \\
& \mathbf{T}_{n}=\frac{\Delta t}{2}\left(\mathbf{I}-\mathbf{B}_{\beta n}\right)^{-1} \mathbf{P}_{32} .
\end{aligned}
$$

The symbol $\mathbf{P}_{32}$ means embedding $\mathrm{R}_{2} \rightarrow \mathrm{R}_{3}$

$$
\mathbf{P}_{32}=\left\|\begin{array}{cc}
\mathrm{O} & \mathbf{1} \\
\mathrm{O} & \mathrm{O} \\
\mathbf{1} & \mathrm{O}
\end{array}\right\|, \quad \mathbf{P}_{23}=\left\|\begin{array}{ccc}
\mathrm{O} & \mathrm{O} & \mathbf{1} \\
\mathbf{1} & \mathrm{O} & \mathrm{O}
\end{array}\right\| \quad \text { is } \quad \mathbf{R}_{3} \rightarrow \mathbf{R}_{2}
$$

projection matrix, $\mathbf{R}_{n}=\left(\mathbf{I}-\mathbf{B}_{\beta n}\right)^{-1}\left(\mathbf{I}+\mathbf{B}_{\beta n}\right)$.

The inverse matrix has the form

$\left.\mathbf{R}_{n}=\frac{2}{d} \| \begin{array}{ccc}0.5\left(1+B_{\beta 1}^{2}-B_{\beta 3}^{2}\right) & B_{\beta 3} & B_{\beta 1} B_{\beta 3} \\ -B_{\beta 3} & 0.5\left(1-\left(B_{\beta 1}^{2}+B_{\beta 3}^{2}\right)\right) & B_{\beta 1} \\ B_{\beta 1} B_{\beta 3} & -B_{\beta 1} & 0.5\left(1-\left(B_{\beta 1}^{2}-B_{\beta 3}^{2}\right)\right)\end{array}\right) \|$,

where $d=1+B_{\beta 1}^{2}+B_{\beta 3}^{2}$.

Then the matrices $\mathbf{T}_{\mathrm{n}}$ and $\mathbf{R}_{\mathrm{n}}$ are written as follows

$$
\mathbf{T}_{n}=\frac{\Delta t}{2 d}\left\|\begin{array}{cc}
B_{\beta 1} B_{\beta 3} & 1+B_{\beta 1}^{2} \\
B_{\beta 1} & -B_{\beta 3} \\
1+B_{\beta 3}^{2} & B_{\beta 1} B_{\beta 3}
\end{array}\right\|,
$$

$\left.\mathbf{R}_{n}=\frac{2}{d} \| \begin{array}{ccc}0.5\left(1+B_{\beta 1}^{2}-B_{\beta 3}^{2}\right) & B_{\beta 3} & B_{\beta 1} B_{\beta 3} \\ -B_{\beta 3} & 0.5\left(1-\left(B_{\beta 1}^{2}+B_{\beta 3}^{2}\right)\right) & B_{\beta 1} \\ B_{\beta 1} B_{\beta 3} & -B_{\beta 1} & 0.5\left(1-\left(B_{\beta 1}^{2}-B_{\beta 3}^{2}\right)\right)\end{array}\right) \|$.

In accordance with the finite-difference scheme (3), an iterative procedure is used to advance the particles in time. This procedure consists of three steps. The first step of prediction is performed in the local auxiliary Cartesian coordinate system. In the time step $t_{n}$, the values $\mathbf{x}_{\mathrm{n}}, \mathbf{v}_{\mathrm{n}-1 / 2}, \mathbf{E}_{\mathrm{n}}, \mathrm{a}_{\mathrm{n}}$ are known, but the field $\mathbf{E}_{\mathrm{n}+1}\left(\mathbf{x}_{\mathrm{n}+1}\right)$ is unknown. Therefore, the first step is performed with the provided electric field $\mathbf{E}_{n+1}^{(0)}\left(\mathbf{x}^{(0)}\right)=\mathbf{E}_{n}\left(\mathbf{x}_{n}\right)$ instead $\mathbf{E}_{n+1}\left(\mathbf{x}_{n+1}\right)$.

$$
\left\{\begin{array}{ccc}
\tilde{\mathbf{v}} & = & \mathbf{T}_{n}\left(c_{q m} \mathbf{E}_{n}\left(\mathbf{x}_{n}\right)+\mathbf{a}_{n-1}\right)+\mathbf{R}_{n} \mathbf{v}_{n-1 / 2} \\
\tilde{\mathbf{x}} & = & \mathbf{x}_{n}+\mathbf{P}_{23} \tilde{\mathbf{v}} \Delta t
\end{array},\right.
$$

where $c_{q m}=q / \mathrm{m}$. This new intermediate position of the particles is not consistent with the electric field $\nabla \cdot \mathbf{E}_{n+1} \neq 4 \pi \tilde{\rho}$, where $\tilde{\rho}$ is a charge density calculated from the intermediate coordinates of the particles. To fix this, you need one or more correction steps, $\mathbf{x}^{(s+1)}=\mathbf{x}^{(s)}+\boldsymbol{\delta} \mathbf{x}^{(s+1)}$, where $\mathrm{s}$ is the iteration number with $\mathbf{x}^{(0)}=\widetilde{\mathbf{x}}$. The difference in the coordinates of the particles is equal to $\delta \mathbf{x}^{(s+1)}=c_{q m} \Delta t \mathbf{P}_{23} \mathbf{T}_{n}\left(\mathbf{E}_{n+1}\left(\mathbf{x}_{n+1}\right)-\mathbf{E}^{(s)}\left(\mathbf{x}^{s}\right)\right)=\mathbf{T}_{22, n} \delta \mathbf{E}^{(s+1)}$,

where

$$
\mathbf{T}_{22, n}=c_{q m} \Delta t \mathbf{P}_{23} \mathbf{T}_{n}=\frac{c_{q m} \Delta t^{2}}{2 d}\left\|\begin{array}{ll}
1+B_{\beta 3}^{2} & B_{\beta 1} B_{\beta 3} \\
B_{\beta 1} B_{\beta 3} & 1+B_{\beta 1}^{2}
\end{array}\right\| .
$$

This discrepancy in the coordinates of the particles can be physically interpreted as the "polarization" of the plasma with the polarization vector $\mathbf{P}^{(s)}=-\nabla \cdot\left(\rho^{(s)} \mathbf{T}_{22, \mathbf{n}}\right)$. 
This leads to an implicit elliptic equation for the electric field divergence $\boldsymbol{\delta} \mathbf{E}^{(s+1)}$

$$
\nabla \cdot \breve{\boldsymbol{\varepsilon}}^{(s)} \boldsymbol{\delta} \mathbf{E}^{(s+1)}=4 \pi \rho^{(s)}-\nabla_{h} \cdot \mathbf{E}^{(s)},
$$

where $\breve{\boldsymbol{\varepsilon}}^{(s)}=\mathbf{I}+4 \pi \boldsymbol{\chi}_{e}^{(s)}, \boldsymbol{\chi}_{e}^{(s)}=\rho_{e}^{(s)} \mathbf{T}_{22, n}$,

$\mathbf{E}^{(s+1)}=\mathbf{E}^{(s)}+\boldsymbol{\delta} \mathbf{E}^{(s+1)}$.

The last final step is required to correct the intermediate speed values

$$
\mathbf{v}_{n+1 / 2}=\widetilde{\mathbf{v}}+\mathbf{T}_{n} c_{q m} \boldsymbol{\delta} \mathbf{E}^{s+1}\left(\mathbf{x}^{s+1}\right)
$$

and particles acceleration

$$
\mathbf{a}_{n}=\frac{1}{2}\left(c_{q m} \mathbf{E}^{(s+1)}\left(\mathbf{x}^{s+1}\right)+\mathbf{a}_{n-1}\right) .
$$

\section{THE FORCES ACTED ON THE CHARGED PARTICLES IN THE TOKAMAK PLASMA}

When the emission of the charged particles from dust grain surface is absent, we use the "Orbital Motion Limited" (OML) model for dust surface charging [8]. In the frame of OML, the surface floating potential $\varphi$ is determined by the electron current $J_{e}$ to the ion current $J_{i}$ equality [9] (Fig. 1), where

$$
\begin{aligned}
& J_{e}=1 / 4 \cdot n_{e 0}\left(8 T_{e} /\left(\pi m_{e}\right)\right)^{1 / 2} \frac{1}{2} \exp \left(e \varphi / T_{e}\right) A_{d} e, \\
& J_{i}=n_{i 0}\left(2 T_{i} / m_{i}\right)^{1 / 2} F_{\Gamma}\left(u, \chi / \delta_{T}\right) \sigma_{d} e .
\end{aligned}
$$

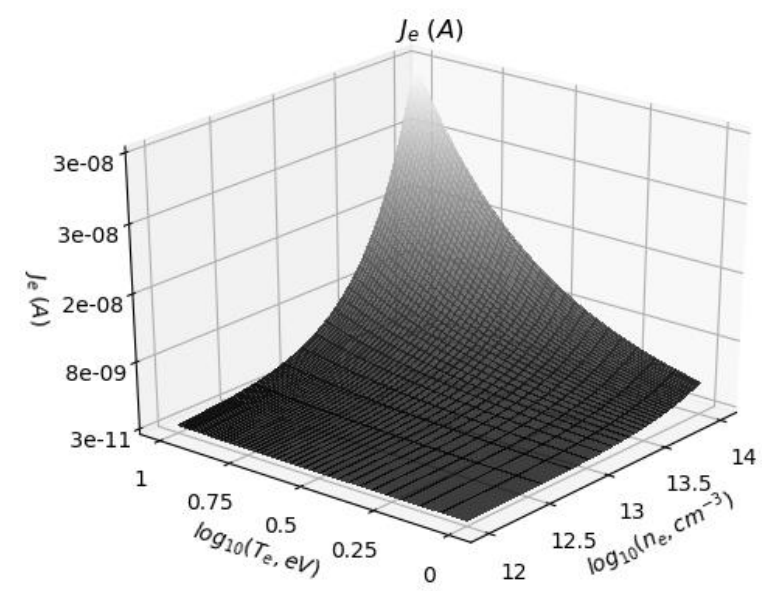

Fig. 1. Electron flux falling on the dust grain surface

Here $n_{e 0}, n_{i 0}$ and $v_{i 0}$ are not disturbed electron and ion densities and plasma velocity far from the dust grain, $\mathrm{m}_{\mathrm{i}}$ is ion mass, $u=v_{i 0}\left[2 T_{i} / m_{i}\right]^{1 / 2}, \quad \delta_{T}=T_{i} / T_{e}, \chi T_{e}=-e$, $\sigma_{d}=\pi R_{d}^{2}$ is a cross section area, and $A_{d}=4 \sigma_{d}$ is a dust grain surface area.

$$
\begin{aligned}
F_{\Gamma}\left(u, \chi / \delta_{T}\right)= & {\left[u+(2 u)^{-1}-1+\chi / \delta_{T} / u\right\rfloor \operatorname{erf}(u)+} \\
& +(\pi)^{-1 / 2}-1 / 2 \exp \left(-u^{2}\right),
\end{aligned}
$$

$F_{\Gamma}\left(u, \chi / \delta_{T}\right)=\left[u+(2 u)^{-1}-1+\chi / \delta_{T} / u\right] \operatorname{erf}(u)+(\pi)^{-\frac{1}{2}}-1 / 2 \exp \left(-u^{2}\right)$, and $\operatorname{erf}(x)$ is error function. It was assumed at deriving of the equations $(9,10)$, that (i) potential is attractive $(<0)$, and axial symmetric, (ii) plasma ions are once ionized, and (iii) $n_{e 0}=n_{i 0}$.
The floating plasma potential is determined by the following transcendent equation $e / T_{e}=-\chi=1 / 2 \ln \left|\left[m_{e} / m_{i}\right] \delta_{T}\right|+\ln \left|\xi_{\sigma} F_{\Gamma}\left(u, \chi / \delta_{T}\right)\right|$, where $\xi_{\sigma}=1 / 2(\pi)^{1 / 2}\left[4 \sigma_{d} A_{d}\right]$. Due to ambipolarity of the plasma flux on a dust grain surface it obtains the negative charge, $-e Z_{d}$. Dust density $n_{d}$ assumed to be small, and it satisfies inequality $\mathrm{Z}_{d} n_{d} \ll n_{e}$. The charge number $Z_{d}$ can be obtained from the relation

$$
e^{2} Z_{d} / R_{d}=\chi T_{e} .
$$

This relation follows from that a conduction sphere charge obey the law $e Z_{d}=C$, where $\chi T_{e}=e$ is the surface potential, and electrostatic capacity of the unit of surface area is $C=R_{d}$.

The assumption, that a dust grain surface does not emit the charged particles, has some restrictions. Under certain conditions, especially under emission data absence, the model gives uncertain value and even sign of a dust grain charge and floating potential. In the case of the emission, a balance of the charged particles fluxes on a surface is $J_{e}=J_{i}+J_{e, p h}+J_{e, s e m}-J_{e, s i m}+J_{e, \text { them }}$, where $J_{e, p h}$ is the electron flux due to photoemission; $J_{e, s e m}, J_{e, s i m}$ and $J_{e, t h e m}$ are the fluxes, associated with the secondary electron and ion emission, and the thermoelectron emission. The secondary electron emission [10] strongly depends on a flux and energy of the falling particles, at the same time a thermo-emission critically depends on a surface temperature and material properties, as determines Richardson-Dushman formulae

$$
J_{e, \text { them }}=A_{d} P_{s b}\left[4 \pi e m_{e}\left(T_{d}\right)^{2} / h^{3}\right] \exp \left(-A_{w} /\left(T_{d}\right)\right) .
$$

$A_{w}$ is a working function, $h$ is the Plank constant, $P_{s b}\left(T_{d}\right)$ is a penetration factor of a potential wall [11] (Fig. 2).

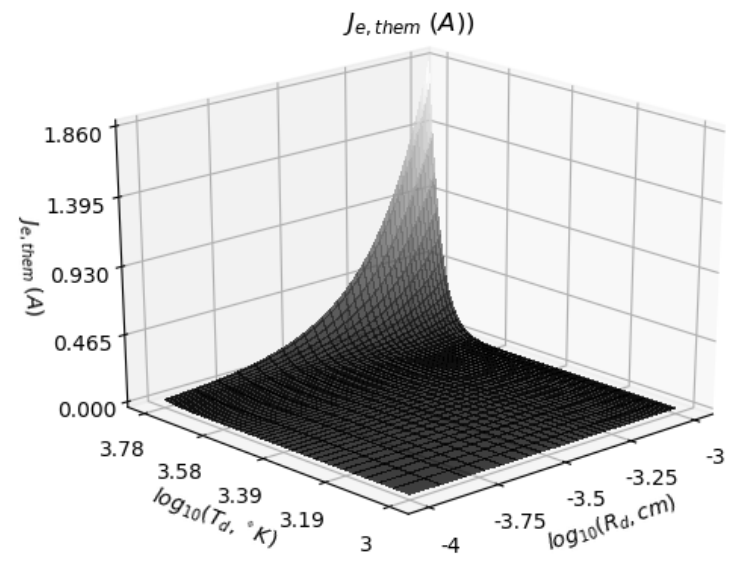

Fig. 2. Electron flux from a dust grain surface as a result of the thermo-electron emission

Under a high temperature of the dust grains, thermoelectron emission may be dominant, which leads to a positive dust grain surface charge. As a result a great flux of the hot electrons amplifies dust grain heating. In so doing the magnetic field and space charge effects may be important. The full force, which acts on a dust grain, is equal to the vector sum $\mathbf{F}_{\mathrm{d}}=\mathbf{F}_{\text {fric, } \mathrm{i}}+\mathbf{F}_{\text {fric, } \mathrm{n}}+\mathbf{F}_{\mathrm{L}}+$ $\mathbf{F}_{\mathrm{g}}$, where $\mathbf{F}_{\text {fric,i }}$ (Fig. 3) is an ion-dust friction force, $\mathbf{F}_{\text {fric,n }}$ is an neutral-dust friction force, $\mathbf{F}_{\mathrm{L}}$ is a Lorentz force, $\mathbf{F}_{\mathrm{g}}$ is a gravity force. 
The following expressions are used for evaluation of these forces

$$
\begin{aligned}
& \mathbf{F}_{\text {fric }, i}=\xi_{i} \xi_{\text {fric }, i} m_{i} n_{i} V_{T i}\left(\mathbf{V}_{i}-\mathbf{v}\right) \sigma_{d}, \\
& \mathbf{F}_{\text {fric }, n}=\xi_{n} \xi_{\text {fric }, n} m_{n} n_{n} V_{T n}\left(\mathbf{V}_{n}-\mathbf{v}\right) \sigma_{d}, \\
& \mathbf{F}_{L}=q \xi_{L}\left(\mathbf{E}_{p l}+\frac{1}{c}[\mathbf{v} \times \mathbf{B}]\right), \\
& \mathbf{F}_{g}=M_{d} \mathbf{g},
\end{aligned}
$$

where $m_{i}, n_{i}, \mathbf{V}_{i}$, and $m_{n}, n_{n}, \mathbf{V}_{n}$ are a mass, a density and a velocity vector of plasma ions and neutral particles correspondingly; $V_{T i}=\left(2 T_{i} / m_{i}\right)^{1 / 2}$ is a thermal velocity of ions, $V_{T n}=\left[2 T n / m_{n}\right]^{1 / 2}$ is a thermal velocity of neutral atoms; $\mathbf{E}_{\mathrm{pl}}$ is a plasma electric field; $\mathbf{B}$ is a magnetic field and $\mathbf{g}$ is a gravity acceleration. The coefficients $\zeta_{i}$, $\zeta_{\mathrm{n}}, \zeta_{\mathrm{L}}$ are the scaling coefficients, which are used for taking in account a change in the force value, if a dust grain is non spherical.

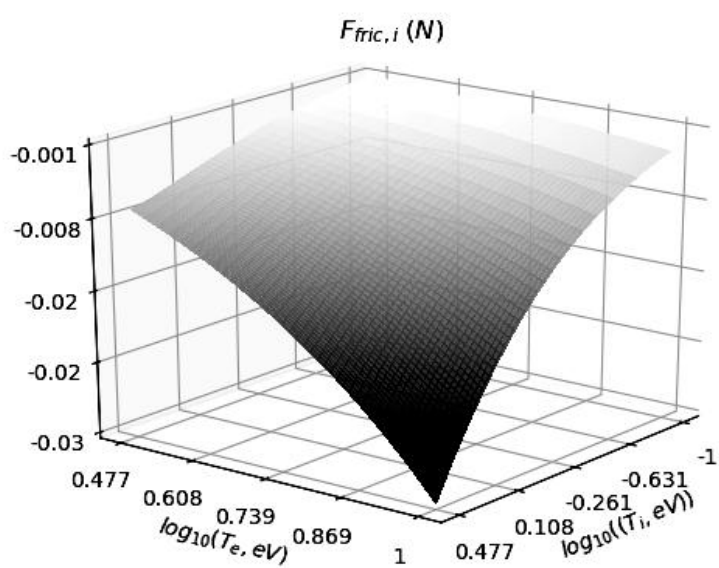

Fig. 3. Plasma ion-dust friction force

Analytic expression for a friction force between a dust grain and plasma ion is found in [6,7]. This force has two components $\mathbf{F}_{\text {fric } \mathrm{n}}=\mathbf{F}_{\text {coll }}+\mathbf{F}_{\text {orb }}$, where $\mathbf{F}_{\text {coll }}$ is a force, caused by an absorption of ions by a dust grain, and $\mathbf{F}_{\text {orb }}$ is a force, caused by the Coulomb scattering. For a negative charged sphere the first component is determined by the expression

$$
\begin{aligned}
\mathbf{F}_{\text {coll }}= & \mathbf{F}_{\text {Epstein }} T_{i} /\left(m_{i} u^{3} \pi^{1 / 2}\right)\left\{u\left[2 u^{2}+1+2 \chi / \delta_{T}\right] \exp \left(-u^{2}\right)+\right. \\
& \left.+\pi^{1 / 2}\left[4 u^{4}+2 u^{2}-1-2\left(1-2 u^{2}\right) \chi / \delta_{T}\right] \operatorname{erf}(u) / 2\right\},
\end{aligned}
$$

where $u=\left|\mathbf{V}_{i}-\mathbf{v}\right| / V_{T i}, \mathbf{F}_{\text {Epstein }}=m_{i} n_{i} V_{T i}\left(\mathbf{V}_{i}-\mathbf{v}\right) \sigma_{d}$.

The second component has a form

$$
\mathbf{F}_{o r b}=2 \mathbf{F}_{\text {Epstein }}\left(\chi / \delta_{T}\right)^{2}(\ln \Lambda) Y(u) / u,
$$

where $Y(u)=\left\{\operatorname{erf}(u)-2 u \pi-1 / 2 \exp \left(-u^{2}\right)\right\} / 2 u^{2}$ is the Chandrasekhar's function; $\left.\ln \Lambda=1 / 2 \cdot \ln \left\{\left(b_{90}\right)^{2}+\left(\eta_{f i t} \lambda_{s}\right)^{2}\right] /\left[\left(b_{90}\right)^{2}+\left(R_{d}\right)^{2}\right]\right\} \quad$ is the Coulomb logarithm; $b_{90}=R_{d} \chi T_{i} /\left\lfloor\delta_{T} m_{i}\left(v_{\text {eff }}\right)^{2}\right\rfloor$ is a sighting parameter, $m_{i}\left(v_{\text {eff }}\right)^{2}=T_{i}\left(3+2 u^{2}\right)$; $\left(\lambda_{s}\right)^{-2}=\left(\lambda_{D}\right)^{-2}\left\{1+\left(3 T_{e} /\left[m_{i}\left(v_{\text {eff }}\right)^{2}\right]\right)\right\}$ is a screening length, $\lambda_{\mathrm{D}}$ is an electron Debye length.

For an exact data reproduction from [6] the parameter $\eta_{f i t}=1+\left(R_{d} / \lambda_{s}\right)\left\{1+\left[T_{e} /\left(6 T_{i}\right)\right]^{1 / 2}\right\}$ is introduced.
This parameter is used in (8), where coefficient of friction $\zeta_{\text {frici }}$ is a function of different plasma parameters and properties of particles.

The friction force $\mathbf{F}_{\text {fric,n }}$ is computed in [12] with the assumption that the cross section of a rigid sphere collisions is a constant and distribution of the neutral atoms is Maxwellian. In this case the coefficient $\zeta_{\text {fricn }}$ in (9) obtains the following view $\left.\zeta_{\text {fric, } n}=\left\{1+s^{2}-(2 s)^{-2}\right\rfloor \operatorname{erf}(s)+\left[s+(2 s)^{-1}\right\rfloor \exp \left(-s^{2}\right) / \pi^{1 / 2}\right\} / s$, where $s=\left|\mathbf{V}_{n}-\mathbf{v}\right| / V_{\mathbf{T} n}$.

\section{DUST PLASMA ION ACOUSTIC INSTABILITY}

Let us consider the dust ion acoustic instability (DIA) at the presence of electromagnetic (EM) waves of large amplitude in a magnetized dust plasma [16, 17] under the following parameters: Ion mass $\mathrm{m}_{\mathrm{i}}=40 \mathrm{amu}$; electron temperature $\mathrm{T}_{\mathrm{e}}=1 \mathrm{eV}$; ion temperature $\mathrm{T}_{\mathrm{i}}=0.1 \mathrm{eV}$; electron-neutral collision cross section $\sigma_{\mathrm{en}}=2 \cdot 10^{-20} \mathrm{~m}^{2}$; ion-neutral collision cross section $\sigma_{\text {in }}=5 \cdot 10^{-19} \mathrm{~m}^{2}$; parameter $\varepsilon Z=0.5$; external electric pumping field $E_{0}=250 \mathrm{~V} / \mathrm{m}$; neutral gas pressure $P=10$ mTorr.

Such parameters were chosen because they are typical for the experiments with dusty plasma.

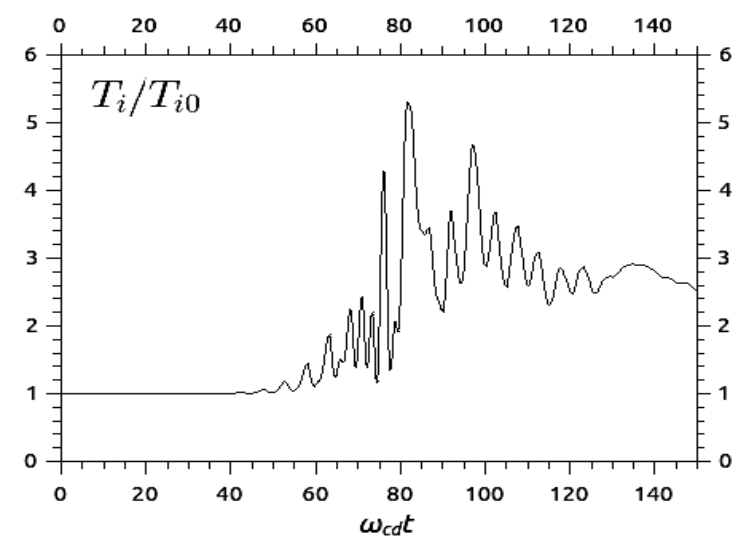

Fig. 4. Evolution of the ion temperature

The results of the computer simulation of the evolution of the dust ion acoustic instability under the action of the external varying electric field are shown in Figs. 4-7. In Fig. 4 ion temperature time dependence is shown. From Fig. 4 it is visible that ion temperature starts oscillate with time and at the simulation time end reaches the level in 1.5 times higher than initial. In Fig. 5 the electric energy density time dependence is shown. The frequency and the growth rate of the electric field energy density in this figure correspond to the doubled frequency and growth rate of the ion acoustic instability of a dust plasma. In Fig. 6 frequency spectrum of the unstable ion acoustic oscillations is shown. In Fig. 7 the ion velocity distribution function is depicted. From this figures it follows, that with time the ion velocity distribution function stops to be Maxwellian. It elongates under the action of the external 
electric field and at last acquires so-called bump on the tail.

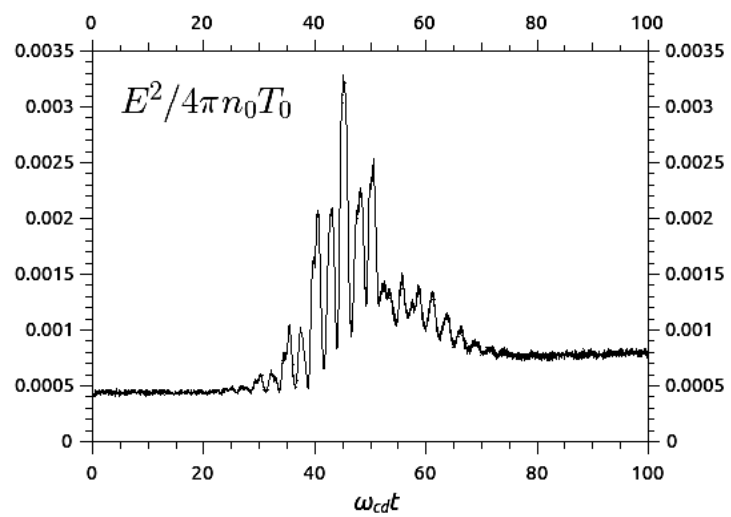

Fig. 5. Electric energy density time dependence

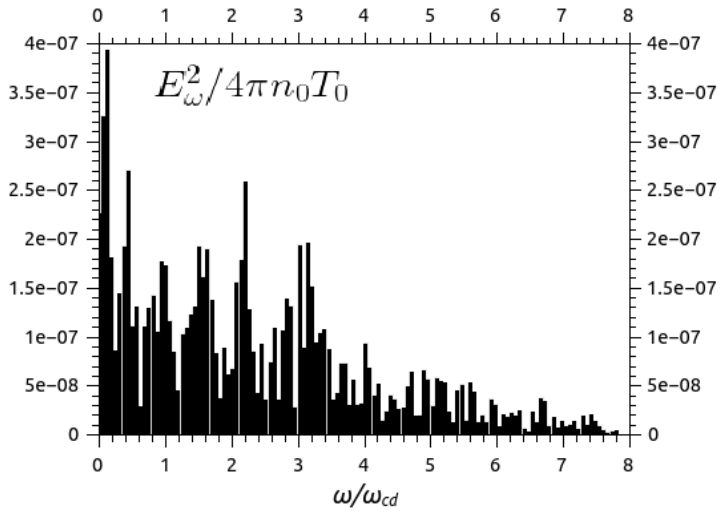

Fig. 6. Frequency spectrum of the unstable oscillations

Thus, under the action of the external electric field on plasma, with neutral gas presence, electron and ion drift appears in the opposite directions. Under the certain conditions this relative drift is sufficient for the ion acoustic waves excitation. The negative charged particles presence facilitates the unstable waves excitation and critic amplitude of the external field for they excitation is decreased when the density of the negative charged dust grains increases.

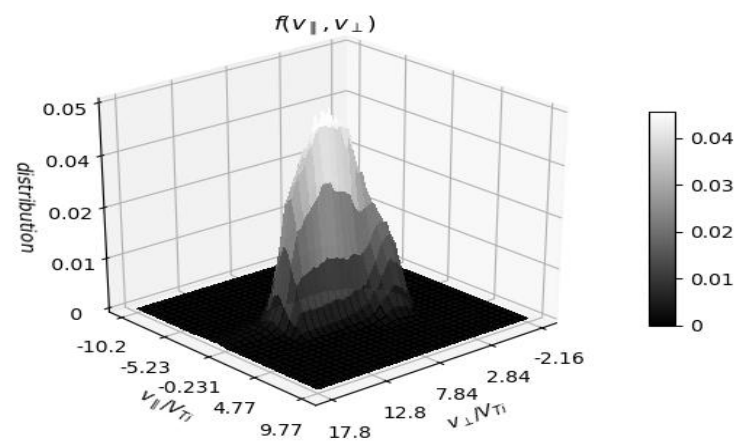

Fig. 7. Ion velocity distribution function

\section{CONCLUSIONS}

The development of ion-acoustic instability in a dusty plasma, turbulence and heating of the magnetized dusty plasma under the action of an external electric field are investigated by the implicit PIC/MCC method of numerical simulation. It is shown that, in an external electric field, the development of ion-acoustic instability is observed, the saturation of which is associated with the capture of plasma ions by the field of the most unstable mode. The presence of a largeamplitude external electric field leads to an increase in the current velocity and the excitation of ion-acoustic instability, the saturation of which is accompanied by stochastization of oscillations and effective heating of the ions of dusty plasma. The relative velocity and temperature of the ions in this case increase significantly. In an alternating electric field, the relative ion velocity oscillates with the frequency of the pumping field. Accordingly, the energy of turbulent ion pulsations and the ion temperatures reach stationary values determined by the amplitude of the pump wave. The ion temperature at the initial stage is permanently increasing. Further, there is a sharp increase in the amplitude of the electric field of unstable oscillations along with a strong rearrangement of their spectrum. Along with the long-wavelength mode, which has the maximum growth rate, the amplitudes of the satellite modes increase. The electric field of the most unstable mode captures ions. It leads to the intensity of turbulent noise decreases, the capture is weakened, the current velocity increases, and instability grows, as a result of which the amplitude of the electric field again increases to values sufficient for the capture of ions. Then there is an increase in the ion temperature and a significant decrease in the current velocity. The ion temperature reaches a stationary level, which is much higher than their initial temperature. The oscillations of ions at this stage of the process are of a stochastic nature, as evidenced by the mixing of particles in the phase plane and a significant broadening of the ion distribution function.

Thus, the final stage of ion-acoustic turbulence of dusty plasma in the presence of an alternating electric field is similar to the final stage of the development of instability with inertial relative motion of ion and electron flows. However, in an alternating electric field, the velocities of charged particles and their relative velocity oscillate, while the levels of turbulent pulsations and the temperature of the ions reach a stationary level.

\section{REFERENCES}

1. R.W. Hockney, J.W. Eastwood. Computer simu lation using particles. «McGraw-Hill»,1981.

2. V.V. Olshansky. Kinetic simulation of low pressure $\mathrm{RF}$ discharge in nonuniform axisymmetric magnetic field. // Problems of Atomic Science and Technology. Series «Plasma Physics» (82). 2012, № 6, p. 77-80.

3. W.J. Goedheer, M.R. Akdim, Yu.I. Chutov // Contrib. Plasma Phys. 2004, v. 44, p. 395-404.

4. J.E. Allen. Probe theory - the orbital motion approach // Phys. Scripta. 1992, v. 45, p. 497-503.

5. J.B. Boris. Relativistic plasma simulation-opti mization of a hybrid code / Proceedings of the Fourth Conference on Numerical Simulation of Plasmas, November 1970.

6. S.A. Khrapak, A.V. Ivlev, G.E. Morfill. Momentum transfer in complex plasmas // Phys. Review E. 2004, v. 70, p. 056405 . 
7. I.H. Hutchinson. Ion collection by a sphere in a flowing plasma: 3. Floating potential and drag force. // Plasma Phys. Control. Fusion. 2005, v. 47, p. 71-87.

8. H.M. Mott-Smith, I. Langmuir. The Theory of Col lec tors in Gaseous Discharges // Phys. Rev. 1926, v. 28, p. 727-763.

9. E.C. Whipple. Potentials of surfaces in space // Rep. Prog. Phys. 1981, v. 44, p. 1197-1250.

10. E.R. Keiter, M.J. Kushner. Plasma transport around dust agglomerates having complex shapes // J. Appl. Physics.1998, v. 83, p. 5670-5677.

11. P.M. Kolesnikov. Elekrodinamicheskoye usko reniye plazmi. M.: «Atomizdat», 1971, s. 1-389 (in Russian).

12. M.J. Baines, I.P. Williams, A.S. Asebiomo. Resis tance to the motion of a small sphere moving through a gas // Mon. Not. R. Astron. Soc. 1965, v. 130, p. 63-74.
13. W. Eckstein. Section 1. Fuel particle balance and dynamics in plasma facing materials. Physical sput tering and reflection processes in plasma-wall interactions // J. Nucl. Materials. 1997, v. 248, p. 1-8. 14. E.W. Thomas, R.K. Janev, J.J. Smith. Scaling of particle reflection coefficients // Nucl. Instrumental Methods B. 1992, v. 69, p. 427-436.

15. P.K. Shukla and L. Stenflo. Modulational Instabilities of Electromagnetic Waves in a CollisionDomi nated Dust-Electron Plasma // IEEE Trans. Plasma Sci. 2001, v. 29, № 2, p. 267-270.

16. P.K. Shukla, A.A. Mamun. Introduction to Dusty Plasma. «IOP Publishing Ltd», 2002, p. 1-275.

17. Robert L. Merlino. Current-Driven Dust IonAcoustic Instability in a Collisional Dusty Plasma. // IEEE Transactions on Plasma Science. 1997, v. 25, № 1 , p. 60-65.

Article received 05.10.2020

\section{ИОННО-ЗВУКОВАЯ НЕУСТОЙЧИВОСТЬ В ПЫЛЕВОЙ ПЛАЗМЕ}

\section{Я.В. Сусаев, В.В. Ольшанский}

Представлены результаты компьютерного моделирования развития неустойчивости ионно-звуковых волн в пылевой плазме, которая состоит из электронов, ионов, массивных заряженных пылинок и нейтральных атомов, с использованием неявного РІС-кода. В этом коде используется неявная схема интегрирования уравнений движения, а конкретно - прямой неявный метод Лэнгдона-Фридмана. Также представлена реализация алгоритма этой неявной схемы.

\section{ІОННО-ЗВУКОВА НЕСТІЙКІСТЬ У ЗАПОРОШЕНІЙ ПЛАЗМІ}

\section{Я.В. Сусаєв, В.В. Ольшанський}

Представлено результати комп'ютерного моделювання розвитку нестійкості іонно-звукових хвиль у запорошеній плазмі, яка складається з електронів, іонів, масивних заряджених порошинок та нейтральних атомів, з використанням неявного РІС-коду. У цьому коді використовується неявна схема інтегрування рівнянь руху, а саме - прямий неявний метод Ленгдона-Фрідмана. Також представлено реалізацію алгоритму цієї неявної схеми. 